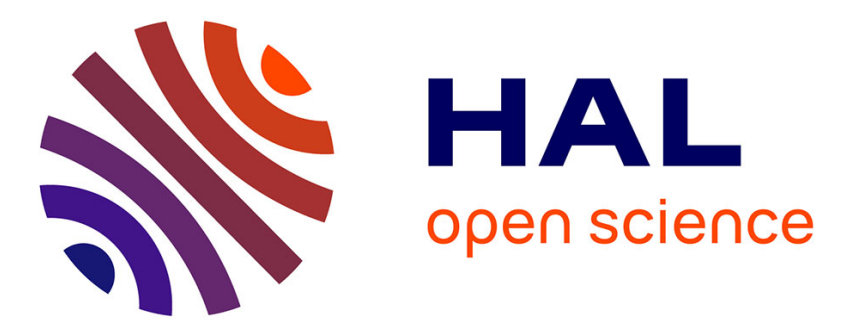

\title{
Measurements of temperature profiles in gases by laser beam deflection
}

\author{
Lin Zhang, Jean-Pierre Petit, J. Taine
}

\section{To cite this version:}

Lin Zhang, Jean-Pierre Petit, J. Taine. Measurements of temperature profiles in gases by laser beam deflection. Revue de Physique Appliquée, 1989, 24 (3), pp.401-410. 10.1051/rphysap:01989002403040100 . jpa-00246062

\section{HAL Id: jpa-00246062 https://hal.science/jpa-00246062}

Submitted on 1 Jan 1989

HAL is a multi-disciplinary open access archive for the deposit and dissemination of scientific research documents, whether they are published or not. The documents may come from teaching and research institutions in France or abroad, or from public or private research centers.
L'archive ouverte pluridisciplinaire HAL, est destinée au dépôt et à la diffusion de documents scientifiques de niveau recherche, publiés ou non, émanant des établissements d'enseignement et de recherche français ou étrangers, des laboratoires publics ou privés. 


\title{
Measurements of temperature profiles in gases by laser beam deflection
}

\author{
Lin Zhang, Jean-Pierre Petit and Jean Taine \\ Laboratoire EM2C du CNRS et de l'ECP, Ecole Centrale des Arts et Manufactures, Grande Voie des Vignes, \\ 92295 Châtenay-Malabry Cedex, France
}

(Reçu le 21 juillet 1988, accepté le 23 novembre 1988)

\begin{abstract}
Résumé. - La détermination des profils de température dans les gaz est liée à la déviation d'un faisceau lumineux due à un gradient de température à pression constante. La géométrie considérée est un cylindre de section arbitraire caractérisé par des génératrices isothermes. On a étudié une méthode d'estimation des paramètres et une méthode dite « de trajectoire parabolique » afin de traiter les résultats expérimentaux. La technique de mesure est ensuite appliquée à la convection naturelle laminaire le long d'une plaque verticale, isotherme dans l'air. Les profils de température déterminés coïncident avec les résultats de la bibliographie avec une précision inférieure à $2 \%$. Cet accord valide la méthode de mesure.
\end{abstract}

\begin{abstract}
The determination of temperature profiles in gases is based on the light beam deflection due to the refractive index gradient induced by a temperature gradient at constant pressure. The geometry considered for the system is a cylinder of arbitrary section characterized by isothermal generative lines. A parameter estimation method and a parabolical trajectory method are considered to treat experimental data. The measurement technique is then applied to laminar natural convection flow along a vertical, isothermal surface in air. The determined temperature profiles agree with classical literature results within $2 \%$. This agreement qualifies our measurement method.
\end{abstract}

\section{Introduction.}

Temperature measurement by optical methods have received a great deal of attention [1-7] since these methods possess considerable advantages such as no disturbance of the temperature field. The optical methods basically yield a refractive index field and the frequently used ones can be divided into two groups: the shadow and schlieren techniques and the interference methods. Many reports in literature on this subject concern the recording of information on the temperature field by photographs (see [8] for a literature survey). In this paper, we present a temperature field measurement technique by laser beam deflection. The system considered is a cylinder of arbitrary section with isothermal generative lines. The optical system consists of a $\mathrm{He}-\mathrm{Ne}$ gas laser and a photodiode position sensor. The deflected laser beam displacements, induced by the variation of refractive index due to non-uniform temperature in the measurement medium, are recorded for different laser beam positions. Two methods for the treatment of experimental data are studied : one is a Parameter Estimation Method, which gives an analytical tem- perature distribution ; another one is a first approximation method called the Parabolical Trajectory Method. Our measurement technique is applied to laminar natural convection flow along a vertical isothermal plate in order to qualify it. Section 2 briefly reviews the principle of light beam deflection. The two methods for the treatment of experimental data are detailed in section 3 . The experimental setup and procedure are described in section 4 . Results on natural convection are reported in section 5 and a discussion follows.

\section{Principle.}

A light beam crossing a medium of inhomogeneous refractive index $n(\mathbf{r})$, is deflected towards the stronger $n$ value region. The differential equation describing the path of the light beam is [9]

$$
\frac{1}{R(\mathbf{r})}=\frac{1}{n(\mathbf{r})} \operatorname{grad}[n(\mathbf{r})] \cdot \mathbf{u}
$$

where $R(\mathbf{r})$ is the curvature radius at point $\mathbf{r}, \mathbf{u}$ is the unit inner normal vector. For pure gases at constant 
pressure $p$, the refractive index is given as a function of temperature by the Gladstone-Dale law [9]

$$
n=1+\frac{K}{T} \quad \text { with } \quad K=\beta \frac{p T_{\mathrm{s}}}{p_{\mathrm{s}}}
$$

where $T_{\mathrm{s}}$ and $p_{\mathrm{s}}$ are respectively the standard temperature and pressure $\left(T_{\mathrm{s}}=273.15 \mathrm{~K}, p_{\mathrm{s}}=1 \mathrm{~atm}\right)$, $\beta$ is the Gladstone-Dale constant, which depends only on the gas nature and light beam wavelength $\lambda$ [10]. In the case of air and for $\lambda=0.6328 \mu \mathrm{m}$ (the wavelength of $\mathrm{He}-\mathrm{Ne}$ laser), $\beta=2.920 \times 10^{-4}$. Equations (1) and (2) show that the light beam trajectory depends on the temperature distribution of the crossed constant pressure gaseous medium, so we can expect to determine the medium temperature field from the observation of a set of beam trajectories.

Let us consider a gas medium which temperature variation is induced by a hot cylindrical surface of arbitrary section. A laser beam is parallel to the cylinder generative line; the plane containing the laser beam and normal to the hot surface at temperature $T_{\mathrm{w}}$ is illustrated in figure 1 . A deflection of the

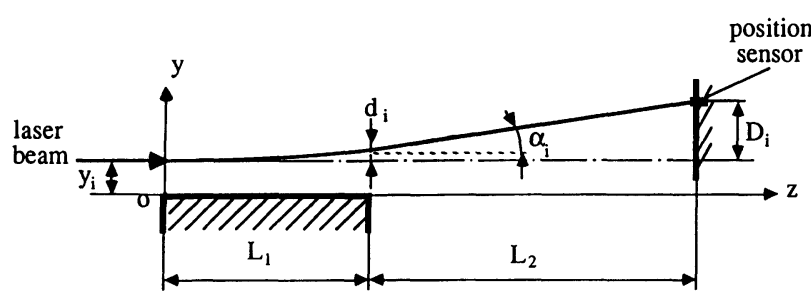

Fig. 1. - Coordinate system in the sectional plane containing light beam and normal to the test surface.

laser beam towards the colder medium region is recorded on a position sensor ; the beam displacement is amplified by the distance $L_{2}$ crossed between the hot surface and the recording location. Assume the generative line of the hot cylinder to be isothermal and length $L_{1}$ large enough for the beam deflection to be mainly due to the gas medium temperature variation in direction $\mathrm{Oy}$. For each $y_{i}$ value, let $d_{i}$ and $\alpha_{i}$ be respectively the laser beam deflection displacement and angle at $z=L_{1}$ (Fig. 1), induced by the one-dimensional temperature variation of the gas medium between $z=0$ and $z=L_{1}$. The recorded beam displacement $D_{i}$ is then expressed by :

$$
D_{i}=d_{i}+L_{2} \tan \alpha_{i}+\Delta_{i}
$$

where $\Delta_{i}$ designates the edges effects correction which will be discussed in section 4 . If the ratio $\frac{L_{2}}{L_{1}}$ is chosen large enough, $d_{i}$ is then much smaller than $L_{2} \tan \alpha_{i}$, the measurements of $D_{i}$ result in the deflection angle $\alpha_{i}$, and the recorded signal $D_{i}$ becomes large; this is helpful for an accurate computer acquisition. The variations of $D$ with $y$ allow us to determine the medium temperature profile as a function of $y$.

\section{Numerical treatment methods.}

Two numerical processing methods have been studied to determine the temperature distribution. The first one is a Parameter Estimation Method (PEM), in which the temperature field is expressed as a given function depending on a few parameters ; the sequential simplex search [11] is then employed to optimize these parameters. The second method is an approximate one ; in this method the assumption that each light beam crosses a constant refractive index gradient path is taken and leads to a parabolical beam trajectory. This method is then called Parabolical Trajectory Method (PTM).

3.1 Parameter Estimation Method (PEM). For $M$ couples of mesures $\left(y_{i}, D_{i}\right)$, we chose a temperature function depending on $N$ parameters $a_{j}(j=1,2, \ldots N)$ to be determined :

$$
T(y)=f\left(y, a_{1}, a_{2}, \ldots, a_{N}\right) .
$$

The number of parameters $N$ is smaller than the number of measures $M$. In each particular problem, we account for the specified temperature conditions in the choice of function $f$.

The sequential simplex search method [11] due to Spendley and co-workers [12] is used to determine the temperature function parameters. The two main quantities are respectively the parameter vector $\mathbf{A}=\left(a_{1}, a_{2}, \ldots, a_{N}\right)$, called the simplex vertex, and the objective function :

$$
\sigma=\sqrt{\frac{1}{M} \sum_{i=1}^{M}\left(D_{i}^{\mathrm{c}}-D_{i}\right)^{2}}
$$

where $D_{i}^{\mathrm{c}}(i=1,2, \ldots, M)$ are displacements calculated by using the parameter vector $\mathbf{A}$, the temperature function (4), and the basic equations (1) and (2) in conditions $i$. For a problem of $N$ parameters to be determined, $N+1$ initial parameter vectors $\mathbf{A}_{k}$ are introduced so that, among them, $N$ parameters are linearly independent. Generally, the first vector $\mathrm{A}_{0}$ is arbitrarily chosen and the other $N$ 's are taken to be :

$$
\begin{aligned}
& \mathbf{A}_{k}=\mathbf{A}_{0}+\left(a_{k 1}, a_{k 2}, \ldots, a_{k N}\right) k=1,2, \ldots, N \\
& \text { with } a_{k j}=p \delta_{k j}+q\left(1-\delta_{k j}\right) \quad j=1,2, \ldots, N
\end{aligned}
$$

where $\delta_{k j}$ designates the Kronecker symbol ; $p$ and $q$ are given by : 


$$
\begin{aligned}
& p=\frac{s}{N \sqrt{2}}(\sqrt{N+1}+N-1) \\
& q=\frac{s}{N \sqrt{2}}(\sqrt{N+1}-1) .
\end{aligned}
$$

Here $s$ is the size of the edge of the simplex. Corresponding to these $N+1$ parameter vectors, $N+1$ objective function values are evaluated. The procedure is to reject the vertex at which $\sigma$ is worst and to replace it by a new vertex obtained from the other $N$ vertices [11, 12]. The new value of the objective function is calculated at this new vertex, and a comparison made of the new value and other $N$ 's. The procedure is repeated until no subsequent adjustment of $\mathbf{A}$ would lead to further improvement. The criterion of no further improvement can be expressed as :

$$
\sqrt{\frac{1}{N+1} \sum_{j=1}^{N+1}\left(\sigma_{j}-\bar{\sigma}\right)^{2}}<\varepsilon \bar{\sigma}
$$

with

$$
\bar{\sigma}=\frac{1}{N+1} \sum_{j=1}^{N+1} \sigma_{j}
$$

where $\varepsilon$ is a small designated value. This is a criterion of mathematical significance : the average of the last $N+1$ parameter vectors is then the optimum of the parameter vector. The physically significant criterion is :

$$
\frac{\sigma}{D_{\max }}<\varepsilon_{\sigma}
$$

where $\varepsilon_{\sigma}$ is the tolerated displacement error: the ratio of the uncertainty of measures $D_{i}$ to the maximum displacement $D_{\max }$.

3.2 Parabolical Trajectory Method (PTM). - Let us consider the case in which the deflected angle $\alpha_{i}$ is small enough for $\tan ^{2} \alpha_{i}$ to be negligible before 1 (Fig. 1). The approximation of constant refractive index gradient is made over the path of the light beam. For the trajectory $i$, we have :

$$
n=n_{i}+c_{i}\left(y-y_{i}\right)
$$

considering

$$
n-1 \sim 3 \times 10^{-4} \ll 1 .
$$

Equation (1) is simplified as

$$
y^{\prime \prime}=c_{i}=\frac{2 d_{i}}{L_{1}^{2}}=\frac{\alpha_{i}}{L_{1}} \quad y_{i} \leqslant y \leqslant y_{i}+d_{i} .
$$

The beam trajectory is then parabolical. Combining equations (3) and (10), we get the constant $c_{i}$ without consideration of the edges effects correction $\Delta_{i}$

$$
c_{i}=\frac{D_{i}}{L_{1}\left(L_{2}+0.5 L_{1}\right)} .
$$

Substituting relation (2) for $n$ in equation (9), we obtain an expression of the temperature distribution versus $y$ :

$$
\frac{1}{T(y)}=\frac{1}{T_{i}}+c_{i}\left(y_{i}-y\right) \quad y_{i} \leqslant y \leqslant y_{i}+d_{i}
$$

where $T_{i}$ is the medium temperature at $y=y_{i}$ in the region near the wall. For a set of measures $i=1,2, \ldots, M$, we have a set of expressions as equation (12). In order to calculate the temperature at any point $y$, we use an average coefficient $c_{i+\frac{1}{2}}=\frac{1}{2}\left(c_{i}+c_{i+1}\right)$ for the range $y_{i} \leqslant y \leqslant y_{i+1} ;$ we have then an equation similar to equation (12) :

$$
\begin{aligned}
\frac{1}{T(y)}= & \frac{1}{T_{i}}+c_{i+\frac{1}{2}}\left(y-y_{i}\right) \\
& \text { for } y_{i} \leqslant y \leqslant y_{i+1} \quad i=1,2, \ldots, M .
\end{aligned}
$$

The temperatures in the range $0 \leqslant y \leqslant y_{1}$ and $y_{M} \leqslant y \leqslant \infty$ are respectively calculated by:

$$
\begin{array}{ll}
\frac{1}{T(y)}=\frac{1}{T_{\mathrm{w}}}+c_{1} y & \text { for } 0 \leqslant y \leqslant y_{1} \\
\frac{1}{T(y)}=\frac{1}{T_{M}}+c_{M}\left(y-y_{M}\right) & \text { for } y_{M} \leqslant y \leqslant \infty
\end{array}
$$

Continuous temperature conditions at points $y_{i}(i=1,2, \ldots, M)$ are used to deduce the values of temperature $T_{i}$ at these points. The temperature gradient at the wall

$$
\left(\frac{\mathrm{d} T}{\mathrm{~d} y}\right)_{\mathrm{w}}=-c_{1} T_{\mathrm{w}}^{2}
$$

can be employed to evaluate the wall heat flux $q_{\mathrm{w}}=-\lambda\left(\frac{\mathrm{d} T}{\mathrm{~d} y}\right)_{\mathrm{w}}$, where $\lambda$ is the medium conductivity.

When the wall heat flux $q_{\mathrm{w}}$ is known instead of the wall temperature $T_{\mathrm{w}}$, the latter can be calculated by :

$$
T_{\mathrm{w}}=\sqrt{c_{1} \frac{\left|q_{\mathrm{w}}\right|}{\lambda}}
$$

3.3 NUMERICAL SIMULATION. - Numerical simulations have been carried out to test the accuracy and the sensibility to measurement errors of the methods developed in this section. For an arbitrary given polynomial temperature distribution :

$$
\begin{array}{rlrl}
\theta(y) & =\frac{T(y)-T_{\infty}}{T_{\mathrm{w}}-T_{\infty}} & \\
& =1-\frac{3}{2} \frac{y}{E}+\frac{1}{2}\left(\frac{y}{E}\right)^{2} \text { for } 0 \leqslant y \leqslant E \\
\theta(y) & =0 \quad \text { for } E<y \leqslant \infty
\end{array}
$$


we solved the trajectory equation (1) and relation (2) for the conditions $T_{\mathrm{w}}=350 \mathrm{~K}, T_{\infty}=300 \mathrm{~K}$, $L_{1}=0.25 \mathrm{~m}, L_{2}=2.24 \mathrm{~m}$ and $E=0.02 \mathrm{~m}$ to obtain a set of simulated displacements $D_{i}$ without considerating edges effects.

The PEM and PTM are applied to the simulated displacements shown in figure 2a. In PEM calculation, a fifth order polynomial has been considered in this example. As the zero displacement at $E=0.02 \mathrm{~m}$, the conditions $T=T_{\infty}, \frac{\mathrm{d} T}{\mathrm{~d} y}=0$ at $y=E$ were accounted for as well as $T=T_{\mathrm{w}}$ at $y=0$. Thus, only three parameters were to be determined instead of six. The temperature profile is expressed as :

$$
\begin{gathered}
T(y)=T_{\mathrm{w}}-\left(T_{\mathrm{w}}-T_{\infty}\right)\left[a_{1}\left(\frac{y}{E}\right)+\right. \\
+a_{2}\left(\frac{y}{E}\right)^{2}+a_{3}\left(\frac{y}{E}\right)^{3} \\
\left.+a_{4}\left(\frac{y}{E}\right)^{4}+a_{5}\left(\frac{y}{E}\right)^{5}\right] \text { for } 0 \leqslant y \leqslant E \\
T(y)=0 \text { for } E<y \leqslant \infty
\end{gathered}
$$

with the relations $a_{4}=5-4 a_{1}-3 a_{2}-2 a_{3}$ and $a_{5}=3 a_{1}+2 a_{2}+a_{1}-4$.

In order to ensure the accuracy of the results, the search stage is repeated three times by decreasing $\varepsilon$ defined in equation (7) and the size $s$ of the side of the equilateral simplex; $\varepsilon$ and $s$ are respectively taken to be 0.01 and 1 for first search stage; $\varepsilon_{\sigma}$ is kept constant at $10^{-6}$. The condition of equation (6) is quickly satisfied. The center of gravity of the final simplex of the first search stage is chosen to form the initial simplex in the new stage by setting this point to a new vertex and generating the other new vertices as in equation (6) with $\varepsilon=0.001$ and $s=0.1$. A third search stage follows with $\varepsilon=$ 0.0001 and $s=0.01$ in a similar manner. The optimum parameters in each search stage and related
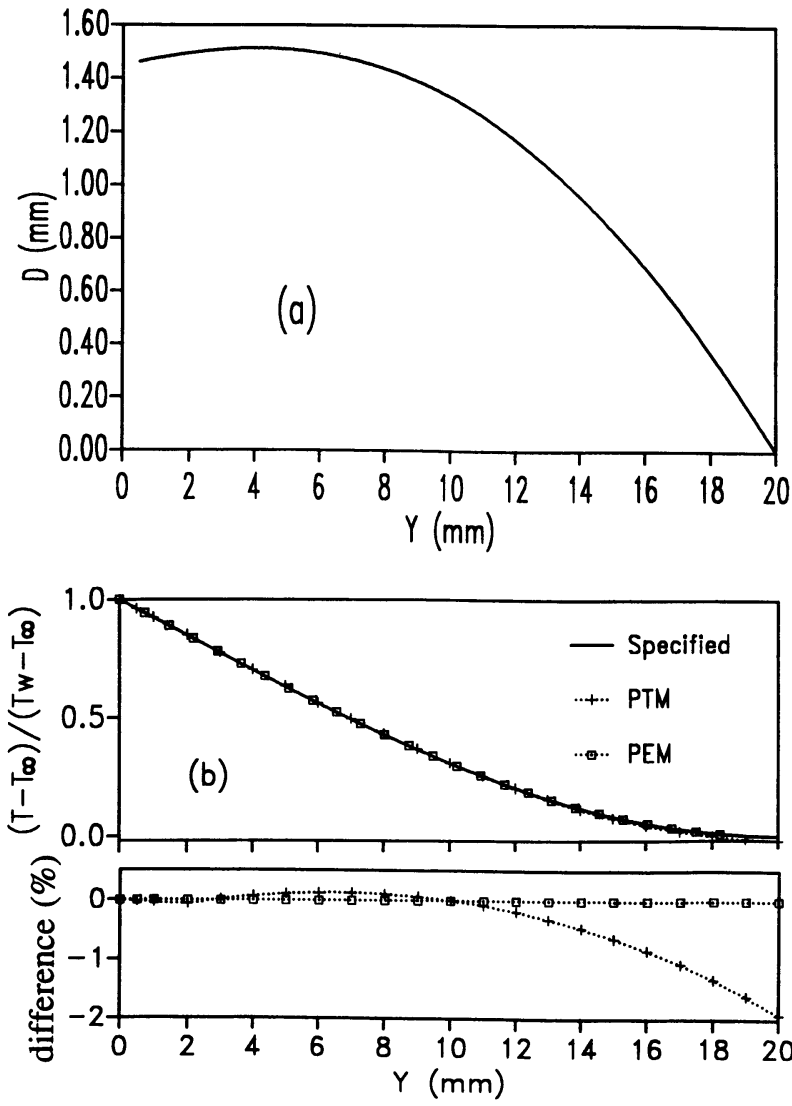

Fig. 2. - (a) Simulated beam deflected displacements, (b) specified non-dimensional temperature profile and the calculated ones, the differences between them in the case of simulation without errors.

values of the objective function are given in table Ia. Accurate polynomial coefficients are obtained after one search stage. In fact, the temperature calculated by the searched polynome is more accurate than the polynomial coefficients. Figure $2 b$ presents, respectively, the temperature profiles obtained with the PEM and PTM, and their deviations from that given

Table I. - Results of three stages of simplex search; polynomial coefficients and related values of the objective function (a) in the case of simulation without errors and (b) in the case of simulation with $10 \%$ errors over the displacements $D$.

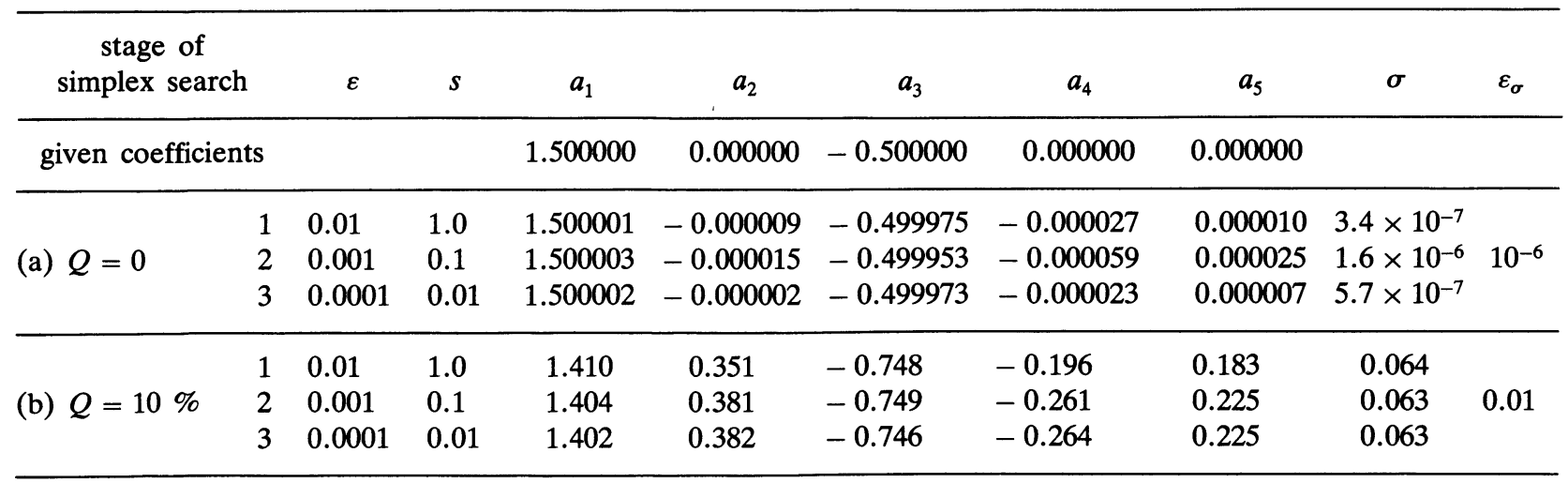


by equation (16). No difference between the given temperature and the results of PEM are observed. The PTM gives a satisfactory temperature in the region near the wall (for small $y$ ), and a value smaller by about $2 \%$ than the given one over the difference $T_{\mathrm{w}}-T_{\infty}$ in the region away from the wall.

A numerical simulation of measurement errors is also carried out. $Q \%$ errors are introduced into the displacements by the following:

$$
D_{i}^{*}=D_{i}+\frac{1}{100} Q D_{1} \omega_{i}
$$

where $D_{i}^{*}$ is the displacement including errors, $D_{i}$ is the one shown in figure $2 \mathrm{a}, \omega_{i}$ is a random function varying between -1 and 1 . An example of the distribution of $D_{i}^{*}$ with $10 \%$ errors is shown in figure 3a (solid line). The PEM and PTM are similarly applied as above, but $\varepsilon_{\sigma}$ is kept to 0.01 . The temperature profiles and the differences between the temperatures form the PEM and PTM and the specified temperature over the difference $T_{\mathrm{w}}-$ $T_{\infty}$ are shown in figure $3 \mathrm{~b}$. The result of the PEM
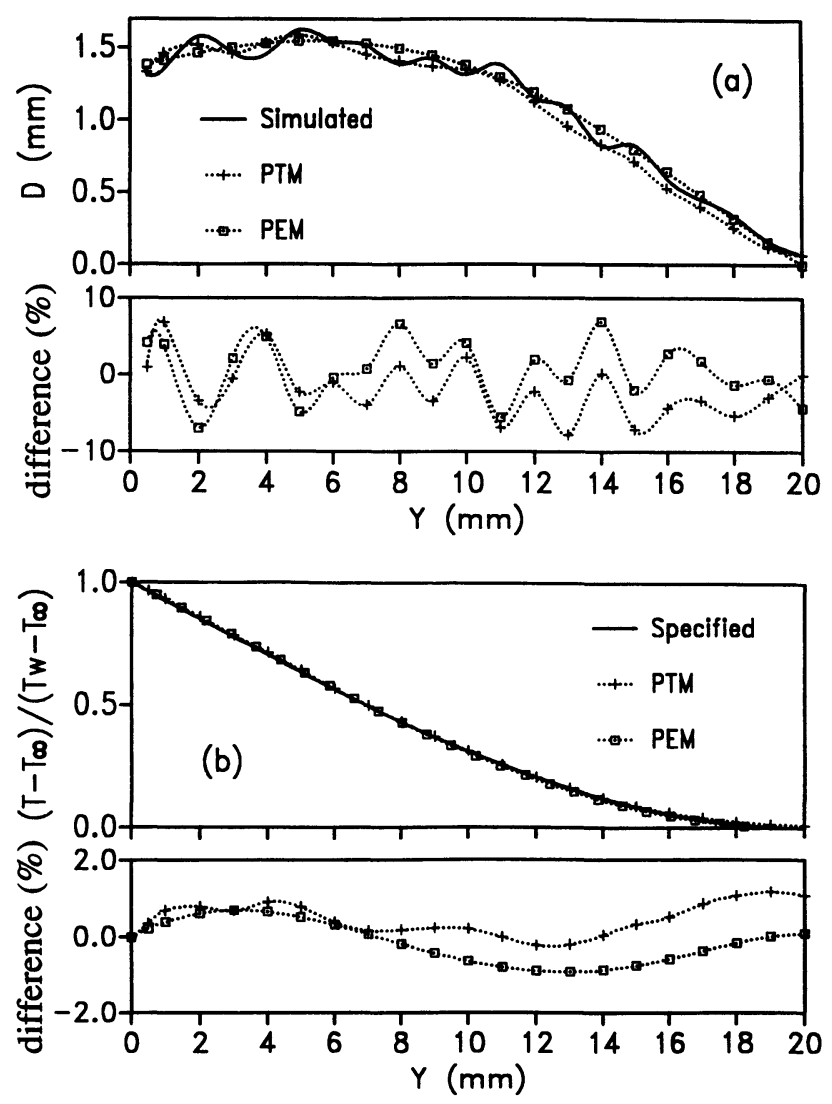

Fig. 3. - (a) Simulated beam deflected displacements and the calculated ones, the differences between them over the maximum simulated displacement, (b) specified non-dimensional temperature profile and the calculated ones, the differences between them in the case of simulation with $10 \%$ errors over the maximum simulated displacement.

REVUE DE PHYSIQUE APPLIQUÉE. - T. 24, N 3, MARS 1989 and the given temperature agree within $1 \%$. Their difference is smaller than the measurement error $(10 \%)$. The conclusions are similar for the PTM. The temperature discrepancies evaluated by this method (less than $2 \%$ ) are also smaller than the measurement errors. The polynomial coefficients and related values of the objective function in the PEM simulations are given out in table Ib. By using the temperature distributions obtained from PEM and PTM, the displacements and the differences between them and the simulated ones over the maximum simulated displacement have been calculated (shown in Fig. 3a). It is clear that the parameter estimation method is much less sensible to the measurement errors than the parabolical trajectory method.

\section{Experimental set-up and procedure.}

The principle and the method described above are applicable to cylindrical surfaces, where the chosen generative line is considered to be isothermal. In order to investigate the feasibility of the method, we have applied it to a laminar natural convection flow along a vertical isothermal flat plate in air; this configuration has been extensively studied and corresponding results are well known [13-17].

A copper vertical flat plate, $1.2 \mathrm{~m}$ high, $0.25 \mathrm{~m}$ wide, $0.01 \mathrm{~m}$ thick, is heated from the back face by twenty electric flat resistances. The test plate thickness and the electric resistance array are carefully determined to get an isothermal test surface. Sixtyfour $1 \mathrm{~mm}$ in diameter chromel-alumel thermocouples are installed in $9 \mathrm{~mm}$ deep holes on the heated face to measure the temperature of the test surface and to test its uniformity. Measurements show that the maximum temperature difference between various points of the test surface is smaller than $2 \%$ of the difference $T_{\mathrm{w}}-T_{\infty}$. The room temperature $T_{\infty}$ is measured by using the same type of thermocouples.

The schematic diagram of the experimental set-up is shown in figure 4. The light source is a SpectraPhysics $0.95 \mathrm{~mW}$ He-Ne laser, which is used in the gaussian mode TEM00. The laser beam is horizontal and kept parallel to the test surface. It can be displaced by using a system of four mirrors and a Micro-Controle step motor driver. A lens is employed to focalize the laser beam near the center of the test surface ; $2.24 \mathrm{~m}$ away from the exit edge of the test surface the laser beam is detected by a SDC photodiode position sensor (type SD-386-2221-251), with an active surface of $10 \times 10 \mathrm{~mm}^{2}$. After natural expansion the laser spot on the position sensor is about 5 to $6 \mathrm{~mm}$ in diameter and is still gaussian. The position sensor is mounted on a Micro-controle step motor driver, used in such a manner that the laser spot is always in the position 


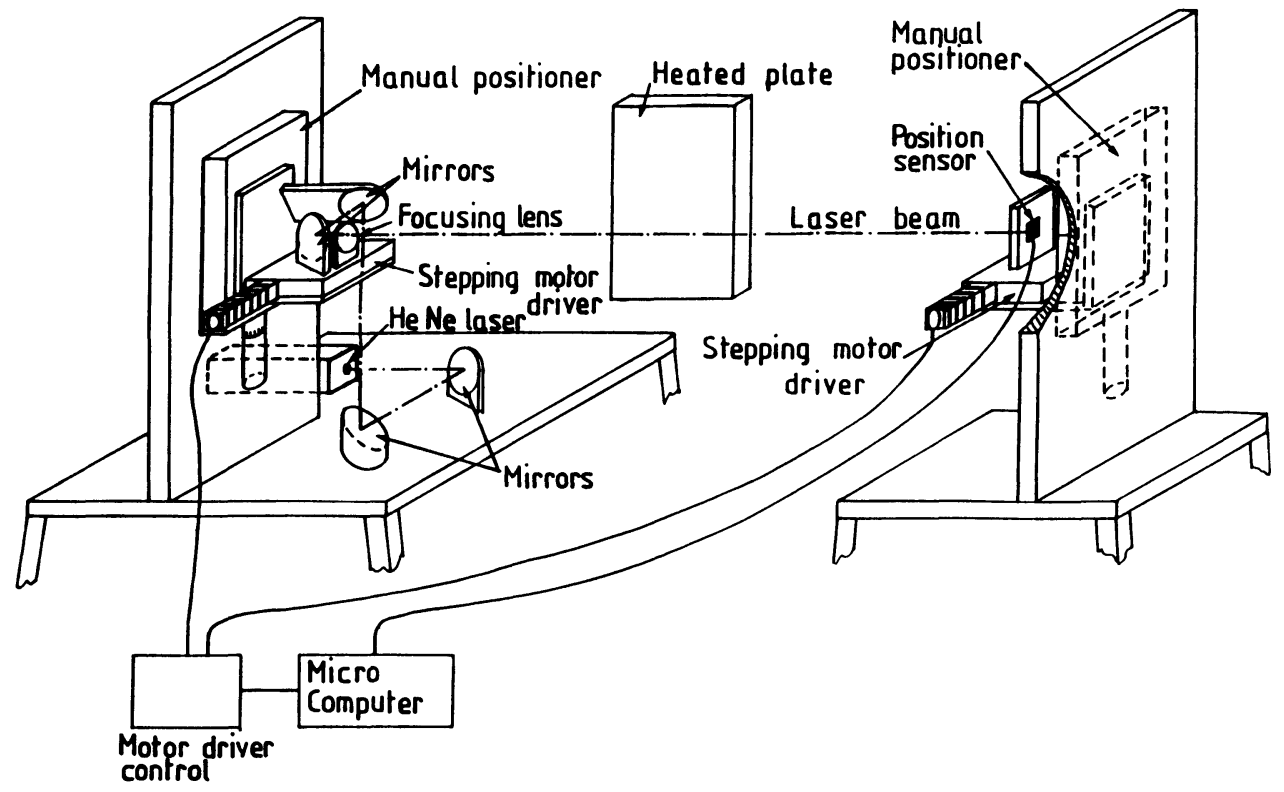

Fig. 4. - Schematic diagram of the experimental set-up.

sensor center region to get optimal response linearity. The response characteristics of the position sensor and its electric amplificator have been studied, the conversion coefficient from the displacement of the center of the laser spot to the acquired electric tension is $C_{\mathrm{de}}=2.80 \pm 0.02 \mathrm{~V} / \mathrm{mm}$ when the center of the laser spot is inside a $4 \mathrm{~mm}$ diameter circular region of the position sensor. During the experiments a drift of the laser spot is expressed in electric tension as $V-V_{0}\left(V_{0}\right.$ and $V$ are respectively the responses before and after the drift). The position sensor is then displaced to follow by the step motor driver the laser spot; this pursuit procedure is repeated once again. After two movements of position sensor, the displacement of the laser spot is determined by the algebraic sum of the imposed total displacement of the position sensor and its measured displacement $C_{\mathrm{de}}\left(V_{\mathrm{L}}-V_{0}\right)$, where $V_{\mathrm{L}}$ is the last response of the position sensor.

The laser beam size and the distance between the test surface and the laser beam axis are difficult to determine accurately. The first one is estimated by the following procedure in the case of a non heated plate : first the laser beam is made to just disappear behind the test surface, then to appear parallel to that by using the step motor driver, until it does not touch any more the test plate. The diameter of the laser beam at the plate edge is estimated by its displacement $\Phi_{\mathrm{L}}=0.90 \pm 0.05 \mathrm{~mm}$. In experiments, the smallest distance $y_{1}$ is chosen to be equal to either $0.55 \mathrm{~mm}$ or $0.60 \mathrm{~mm}$. The precision on $y_{i}(i=1,2, \ldots, M)$ is about $0.05 \mathrm{~mm}$.

The movement of the step motor drivers are controled by an Apple microcomputer, which also performs the acquisition of the laser spot displace- ment. Extensive test and analysis of the experimental set-up and computer controlling program have been carried out for both heated and non heated plate. Measurements of the deflected displacement $D_{i}$ versus $y_{i}$ are automatically started in a still closed room five minutes after the launching of the controlling program. In the non heated case, we have noticed a displacement up to $0.23 \mathrm{~mm}$. In fact, the characteristics of the step motor driver indicate a positioning accuracy of $10 \mu \mathrm{m}$ and a deviation angle of $\pm 5 \times 10^{-5} \mathrm{rd}$, which correspond to a displacement of $\pm 0.15 \mathrm{~mm}$ at a distance of $3 \mathrm{~m}$ (the distance between the motor driver displacing the laser beam and the position sensor). An example of the distribution of $D$ versus $y$ is shown in figure 5 , this distribution is repeatable with an accuracy of $\pm 10 \mu \mathrm{m}$; it will be used for the correction of measures in the hot cases.

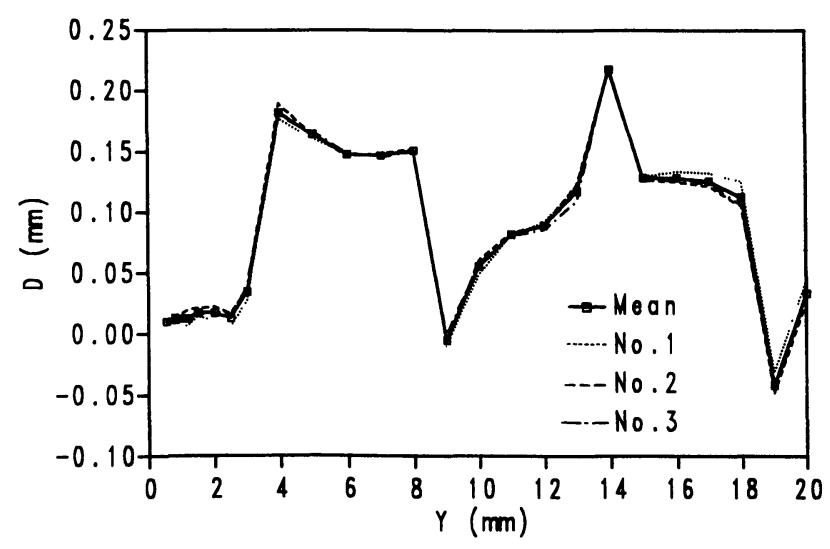

Fig. 5. - Beam displacement distribution due to the measurement system in the case of non-heated plate. 


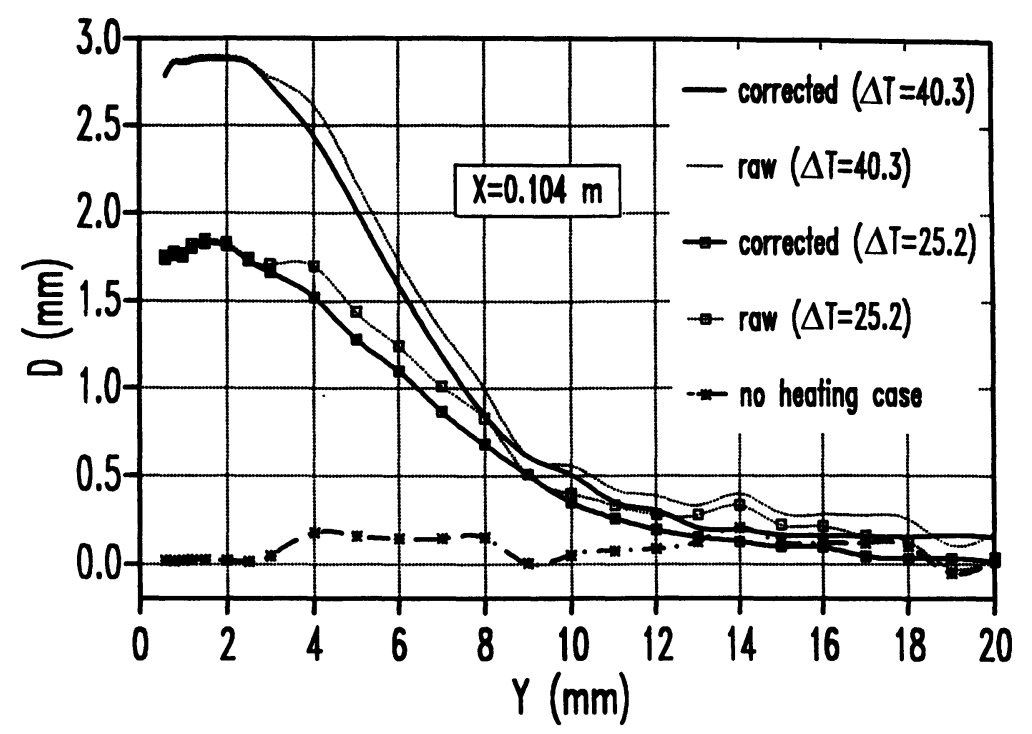

Fig. 6. - Raw and corrected beam deflected displacements. .

Raw and corrected deflected displacements $D$ are given out in figure 6 for two heating cases. The corrected displacements are obtained from the relation :

$$
D=D_{\text {raw }}-D_{0}
$$

where $D_{\text {raw }}$ designates the raw experimental data in the hot case and $D_{0}$ is the mean displacement in the cold case. Figure 6 shows the raw data and the curve smoothed by the correction.

Preliminary experiments have been carried out for plates of different width $(0.25 \mathrm{~m}, 0.30 \mathrm{~m}$ and $0.60 \mathrm{~m}$ ). It was shown that results obtained for the temperature field in the vicinity of the test surface were not disturbed by the edge effects. The analysis of the results in section 5 will lead to the same conclusion. An effect more important than the previous one has to be eliminated : it is the cumulated deflection due to the very small variation of temperature and to ambient air movements over more than two meters of laser beam path to the position sensor. A $0.1 \mathrm{~m}$ diameter PVC pipe has been horizontally hung over this path to stop ambient air movement. Measurements on natural convection have been carried out in a still room.

\section{Results and discussion.}

Measurements of deflected displacement $D$ versus $y$ are carried out for different abscissae $x$ (from 0.03 to $0.58 \mathrm{~m}$ ) from the leading edge and for different wall temperature $T_{\mathrm{w}}$ (from 30 to $63^{\circ} \mathrm{C}$ ). The recorded maximum deviations for the given values of $x$ and $T_{\mathrm{w}}$ vary from 0.73 to $3.84 \mathrm{~mm}$; the related $y$ values are located in the range of 1 to $3 \mathrm{~mm}$.

Three examples of the deflection displacement $D$ and non-dimensional temperature profiles are illustrated in figures 7-9. The Grashof number intro- duced in these figures is a dimensionless parameter defined as [17]

$$
\mathrm{Gr}(x)=\frac{g \beta\left(T_{\mathrm{w}}-T_{\infty}\right) x^{3}}{\nu^{2}}
$$
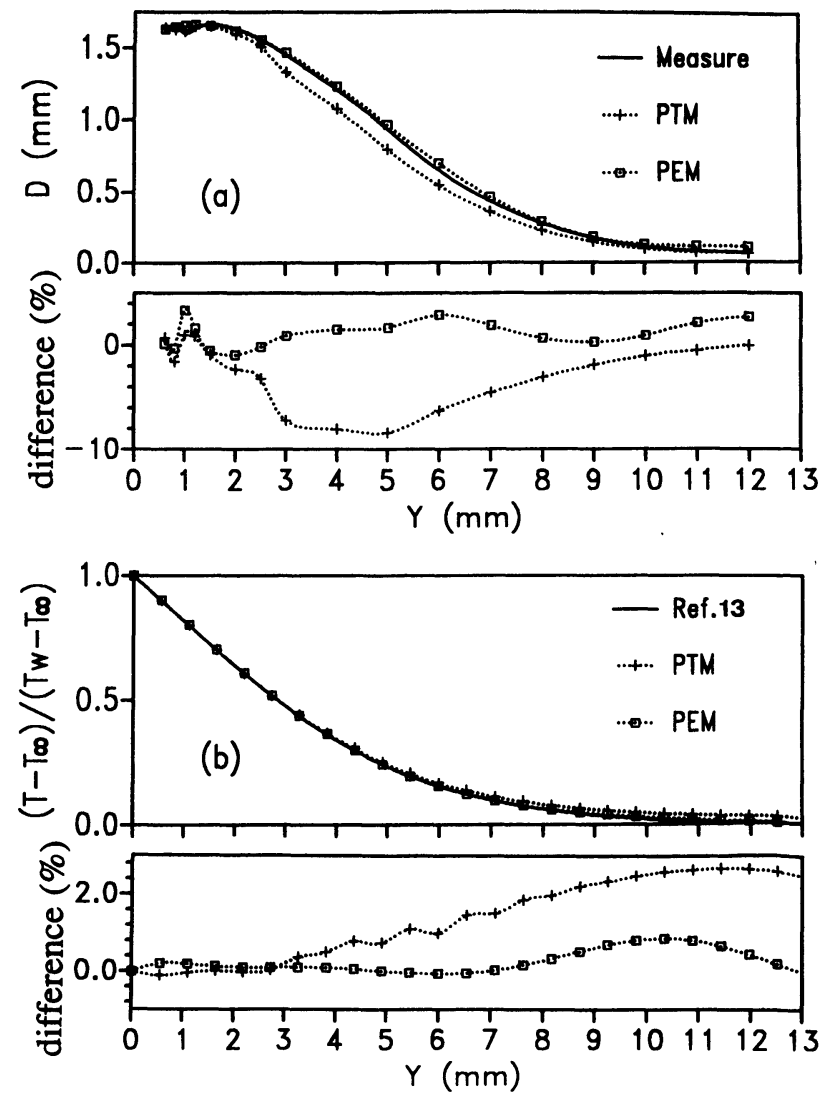

Fig. 7. - (a) Experimental displacements, the ones calculated by the PEM, PTM formulations and its related differences, (b) non-dimensional temperature profiles from Ostrach analysis [13], the PEM and PTM calculations and its related differences in the case of $T_{w}-T_{\infty}=$ $40.7 \mathrm{~K}, x=0.060 \mathrm{~m}, \mathrm{Gr}=9.5 \times 10^{5}$. 

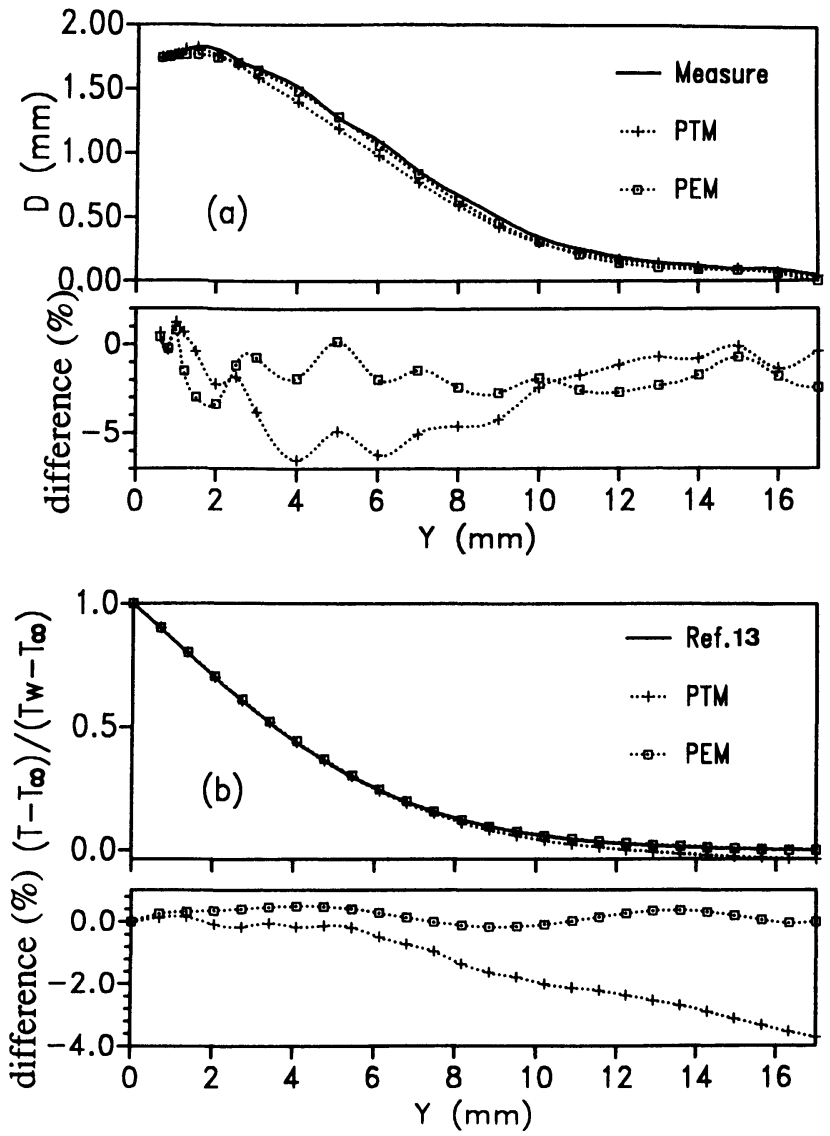

Fig. 8. - (a) Experimental displacements, the ones calculated by the PEM, PTM formulations and its related differences, (b) non-dimensional temperature profiles from Ostrach analysis [13], the PEM and PTM calculations and its related differences in the case of $T_{w}-T_{\infty}=$ $25.1 \mathrm{~K}, x=0.104 \mathrm{~m}, \mathrm{Gr}=3.5 \times 10^{6}$.

We applied the parameter estimation method (PEM) and the parabolical trajectory method (PTM), described in section 3, respectively to the corrected experimental data represented by the solid line in figures 7a-9a; the obtained temperature profiles are shown in figures $7 b-9 b$, in the non dimensional form. The PEM results show excellent agreement with the theoretical laminar natural convection temperature profile, which is calculated by using the Ostrach analysis [13] with constant air properties evaluated at mean temperature $T_{\mathrm{m}}=$ $\frac{\left(T_{\mathrm{w}}+T_{\infty}\right)}{2}$. The maximum dimensionless temperature differences (Figs. $7 \mathrm{~b}-9 \mathrm{~b}$ ) are under $2 \%$. It is observed that the temperature profiles of PTM agree very well with the other two in the vicinity of the wall, but are less accurate in the region far away from the wall. This was expected however since a constant refractive index gradient was assumed over the path of the laser beam in the PTM calculation; as the temperature at point $y_{i}$ is evaluated from all the measures between this point and the wall, all errors accumulate.
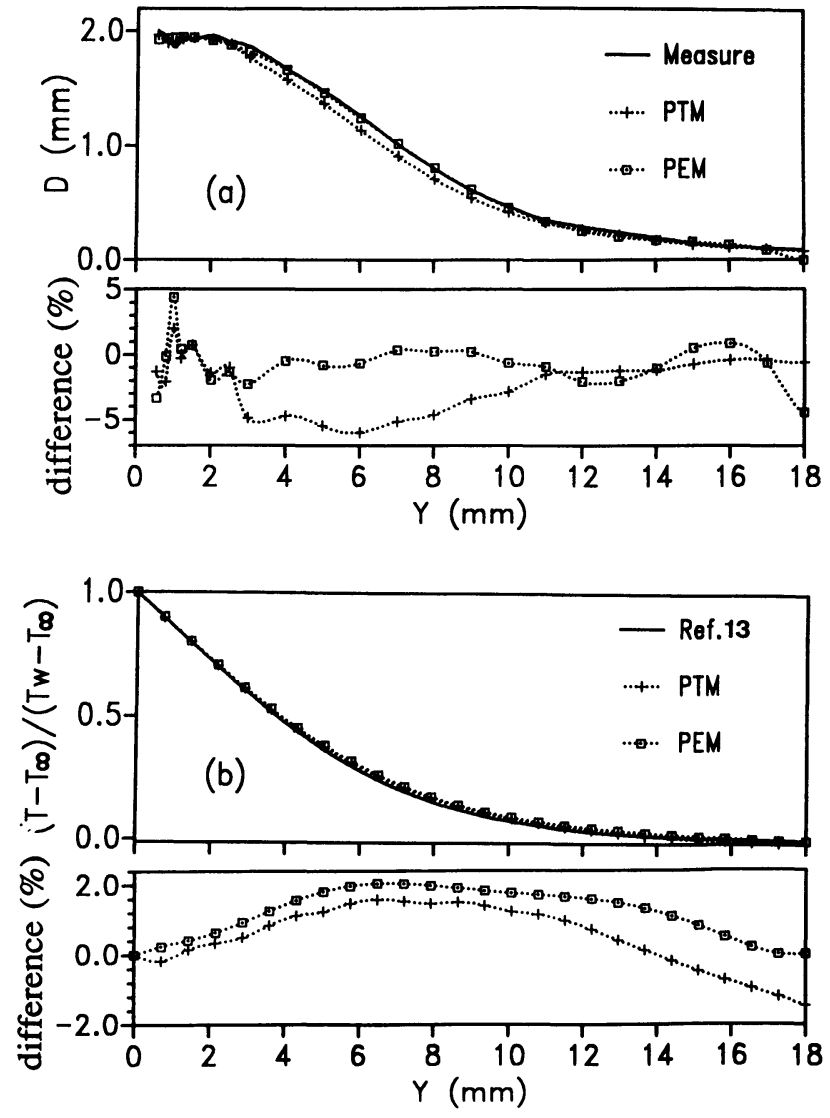

Fig. 9. - (a) Experimental displacements, the ones calculated by the PEM, PTM formulations and its related differences, (b) non-dimensional temperature profiles from Ostrach analysis [13], the PEM and PTM calculations and its related differences in the case of $T_{\mathrm{w}}-T_{\infty}=$ $30.9 \mathrm{~K}, x=0.150 \mathrm{~m}, \mathrm{Gr}=1.2 \times 10^{7}$.

By using the temperature distributions obtained from the PEM and PTM calculations, the displacements are calculated and shown in the same figures as the experimental data (Figs. 7a-9a). The differences between the calculated displacements and the measured ones over the maximum measures of displacement are also presented in figures 7a-9a. Agreement between experimental data and values calculated by the PEM is observed. The temperature distributions of the PTM lead to correct displacement distributions near the wall, but to some discrepancies with both experimental data and PEM results away from the wall.

The polynomial temperature function determined by the PEM has a form similar to equation (17), the geometrical length $E$ corresponds to the experimental uncertainty over displacement $D$; the value of $E$ is evaluated from the value of $y_{i}$ at which $D_{i}$ is smaller than the uncertainty of the reproducible displacement distribution in the case of the nonheated plate. Polynomial coefficients related to figures 7-9 are given out in table II. It is worth noticing that the polynomial temperatures deter- 
Table II. - Polynomial temperature coefficients determined by the simplex search method in the three heating cases as figures 7-9.

\begin{tabular}{lcccccccccc}
\hline & $T_{\mathrm{w}}\left({ }^{\circ} \mathrm{C}\right)$ & $T_{\mathrm{w}}-T\left({ }^{\circ} \mathrm{C}\right)$ & $x(\mathrm{~m})$ & $\mathrm{Gr}(x)$ & $E(\mathrm{~mm})$ & $a_{1}$ & $a_{2}$ & $a_{3}$ & $a_{4}$ & $a_{5}$ \\
\hline Fig. 7 & 60.6 & 40.7 & 0.060 & $9.5 \times 10^{5}$ & 13.6 & 2.40 & 1.67 & -10.62 & 11.63 & -4.08 \\
Fig. 8 & 43.6 & 25.1 & 0.104 & $3.5 \times 10^{6}$ & 17.0 & 2.40 & 1.45 & -9.68 & 10.41 & -3.58 \\
Fig. 9 & 51.0 & 30.9 & 0.150 & $1.2 \times 10^{7}$ & 18.0 & 2.42 & 0.96 & -8.58 & 9.61 & -3.41 \\
\hline
\end{tabular}

mined by the PTM, generally, do not depend on the value of $E$ but on the ratio of $\frac{a_{n}}{E^{n}}$.

In natural convection applications, an important quantity is the dimensionless convection heat transfer coefficient called Nusselt number [17]

$$
\mathrm{Nu}(x)=\frac{h x}{\lambda}=\frac{x}{T_{\mathrm{w}}-T_{\infty}}\left(-\frac{\mathrm{d} T}{\mathrm{~d} y}\right)_{\mathrm{w}}
$$

where $h$ and $\lambda$ are respectively the dimensional convection coefficient and the fluid conductivity ; $x$ is a characteristic geometrical length, which is the abscissa from the leading edge in the case of the natural convection along a vertical plate. In the PEM and PTM calculations, the Nusselt number is respectively given by :

$$
\begin{aligned}
& \mathrm{Nu}(x)=\frac{x a_{1}}{E} \\
& \mathrm{Nu}(x)=\frac{x T_{\mathrm{w}}^{2}}{T_{\mathrm{w}}-T_{\infty}} \frac{D_{1}}{K L_{1}\left(L_{2}+0.5 L_{1}\right)} .
\end{aligned}
$$

The PTM formulation shows that the Nusselt number depends only on the first measure $D_{1}$, this fact allows a fast determination of heat transfer coefficient. Theoretically $\mathrm{Nu}(x)$ would be accurately deduced from the displacement $D_{0}$ related to $y_{0}=0$, but this is limited by the finite diameter of the laser beam. An advantage of the PTM relation (21b) is that $\mathrm{Nu}(x)$ does not explicitly depend on the value of $y_{1}$, although the uncertainty on $y_{1}$ is larger than on the measure of $D_{1}$. The local Nusselt number versus the Grashof number is shown in figure 10 . The solid line represents a natural convection correlation obtained from Ostrach theory [13] for laminar flow on a vertical, isothermal surface in air $(\mathrm{Pr}=0.708)$

$$
\mathrm{Nu}(x)=0.355[\mathrm{Gr}(x)]^{0.25} .
$$

The « best fit " correlations through the PEM and PTM data, shown in figure 10, are respectively

$$
\begin{array}{ll}
\mathrm{Nu}(x)=0.353[\mathrm{Gr}(x)]^{0.25} & (\mathrm{PEM}) \\
\mathrm{Nu}(x)=0.366[\mathrm{Gr}(x)]^{0.25} & (\mathrm{PTM}) .
\end{array}
$$

The heat transfer correlations from the PEM and PTM and theoretical results agree within $0.6 \%$ and less than $3 \%$, respectively. As we have remarked on

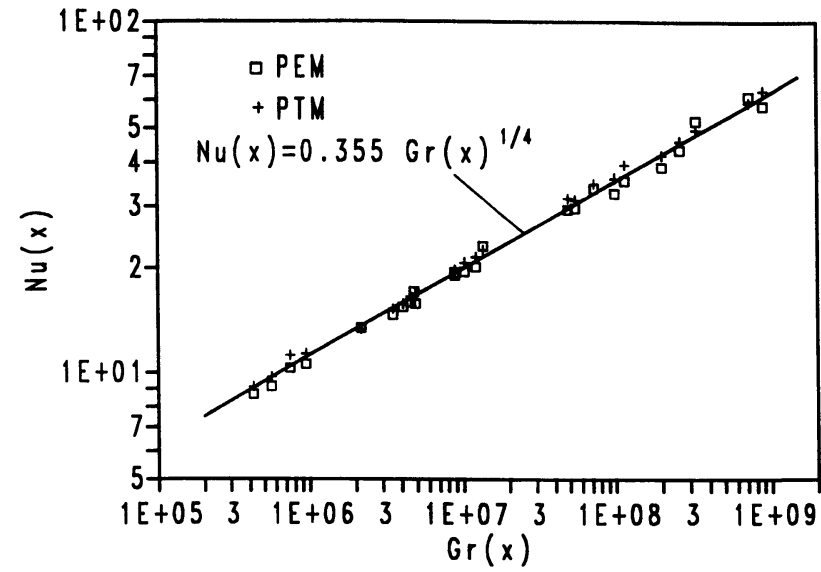

Fig. 10. - Variation of local Nusselt number $\mathrm{Nu}(x)$ with local Grashof number $\mathrm{Gr}(x)$ for air $(\operatorname{Pr}=0.708)$ : theoretical results and our experimental results evaluated by the PEM and PTM.

the deflection displacement distribution, the maximum beam displacement is located near the surface. The first measure, which has been used to calculated $\mathrm{Nu}(x)$ by the PTM, is slightly larger than the beam displacement $D_{0}$, consequently the PTM slightly over-estimates the heat transfer coefficient.

It should be pointed out that our temperature profile and heat transfer coefficient measurement method would be easily applied to any cylindrical system in which the temperature along the generative line can be considered constant. Two examples are illustrated in figure 11 .

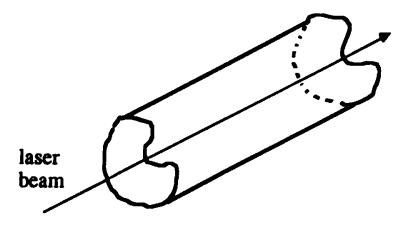

(a)

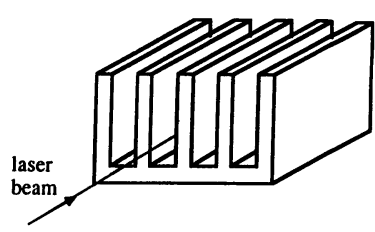

(b)
Fig. 11. - Two examples of the system geometry to which our measurement technique is applicable.

\section{Conclusion.}

A method of determination of the temperature profile and heat transfer rate by laser beam deflection has been developped and applied to a laminar 
natural convection flow on a vertical isothermal flat plat in air. Two numerical processing methods have been tested. A Parameter Estimation Method yields satisfactory temperaure profile and heat transfer coefficients. An approximate one - Parabolical Trajectory Method - gives acceptable temperature profiles near the surface, and can be used for a fast determination of the heat transfer coefficient. The good agreement between our results and classical literature results qualifies our beam deflection method : for laminar natural convection flow along a vertical isothermal plate, the determined temperature profile agrees with literature within $2 \%$, and $1 \%$ for the Nusselt number.

References

[1] HAUF W. and Grigull U., Optical methods in heat transfer, Ad. Heat Transfer (Academic Press, New York) 6 (1970) 133.

[2] Grosjean D. F., Sarka B. and Goss L. P., Opt. Lett. 10 (1985) 324.

[3] Keren E., Bar-Ziv E., Glatt I. and KafRi O., Appl. Opt. 20 (1981) 4263.

[4] Kang Y., Nishino J., SuzUKi K. and Sato T., Flow Visualization II, Hemisphere (Washington) 1982, 77.

[5] Boylan D. E., Carver D. B., Stallings D. W. and Trimmer L. L., AIAA Pap. (1978) 78.

[6] Jacson D. A., Akhavan Leilabady P., Jones J. D. C. and Corke M., J. Phys. E 19 (1986) 143.

[7] VAN Buren P. D. and Viskanta R., Int. J. Heat Mass Transfer 23 (1980) 568.

[8] MerzKirch W., Proc. 7th Int. Heat Transfer Conf. (München) 1 (1982) 91.
[9] BoRn M. and Wolf E., Principles of Optics (Pergamon Press, Oxford) 1975.

[10] Erickson K. E., J. Opt. Soc. Am. 52 (1962) 777.

[11] BEVERIDGE G. S. G. and SCHECHTER R. S., Optimization : theory and practice (McGraw-Hill Book Company, New York) 1970.

[12] SPENDleY W., HeXT G. R. and Himsworth F. R., Technometrics 4 (1962) 441.

[13] Ostrach S., NACA Rep. 1111 (1953).

[14] LeFevre E. J., Proc. Int. congr., 9th (Brussel) Appl. Mech. 4 (1956) 168.

[15] Kuiken H. K., J. Eng. Math. 2 (1968) 355.

[16] Kuiken H. K., J. Fluid Mech. 37 (1969) 785.

[17] Kays W. M. and Crawford M. E., Convective heat and mass transfer (McGraw-Hill Book Company, New York) 1980. 